\title{
Patterns of spinal cord injury in automobiles versus motorcycles and bicycles
}

\author{
Omar Medina ${ }^{1} \cdot$ Varun Singla $^{1} \cdot$ Charles Liu $^{2} \cdot$ Dudley Fukunaga ${ }^{2} \cdot$ Kevin Rolfe ${ }^{1,2}$
}

Received: 6 April 2020 / Revised: 29 July 2020 / Accepted: 30 July 2020

(c) The Author(s), under exclusive licence to International Spinal Cord Society 2020

\begin{abstract}
Study design Retrospective case series.

Objectives To examine the patterns and relative rates of occurrence of spinal cord injury (SCI) in automobiles compared to motorcycles and bicycles.

Setting Los Angeles County, California.

Methods A retrospective chart review of SCI consults at Rancho Los Amigos National Rehabilitation Center in Los Angeles County, California between 2003 and 2013 were selected and screened for a mechanism of injury involving a vehicular accident. Chart review was performed to determine neurological levels and extent of impairment, which were graded according to the International Standards for Neurological Classification of Spinal Cord Injury.

Results We identified 398 cases of SCI from 2003 to 2013 that fit the inclusion criteria. Overall, the relative percentages of ASIA impairment scale (AIS) A/B/C/D did not differ statistically across automobiles, motorcycles, or bicycles. When stratified by spinal region, motorcycles had a higher percentage of thoracic SCIs compared to automobiles. Automobiles resulted in more cervical SCIs with few injuries in the lumbar region. Bicycle patterns followed automobiles, not motorcycles. Thoracic SCIs were more likely graded motor complete than cervical or lumbar injuries, regardless of the mechanism.

Conclusions Automobile, motorcycle, and bicycle related SCIs occur primarily in the cervicothoracic region. SCIs due to motorcycle accidents have a higher predilection for the thoracic region, and there is a statistically higher percentage of motor complete injuries. A higher percentage of cervical SCIs occur as a result of automobile and bicycle accidents. Extrapolations from motor vehicle usage data suggest that the relative rate of occurrence of SCI for motorcycles is higher than for automobiles.
\end{abstract}

\section{Introduction}

Motor vehicle collisions are a serious societal problem and one of the leading causes of morbidity, disability, and mortality. Although not in the top 10 causes of death in 2015, they ranked 7th in years of life lost, demonstrating how they disproportionately affect younger age groups [1, 2]. Per Hall et al., 445,911 disability-adjusted life years resulted from spinal cord injury (SCI) in the United States in 2010, placing

Varun Singla

vsingla945@gmail.com

1 Department of Orthopaedic Surgery, Harbor-UCLA Medical Center, Torrance, CA, USA

2 Department of Neurosurgery and Orthopedic Surgery, Rancho Los Amigos National Rehabilitation Center, Downey, CA, USA the national burden of SCIs above HIV and AIDS [3]. Motor vehicle collisions are also the leading cause of SCI, accounting for almost $40 \%$ of new SCIs each year [4].

While standard passenger vehicles make up a large majority of these injuries and fatalities in the USA, motorcycles are becoming an increasingly popular choice of transportation, with the national number of registered motorcycles climbing each year. There were $8,679,380$ registered motorcycles in 2016 in the USA, a $21.6 \%$ increase since 2007. With this rise in the number of motorcycles on the road has come a concurrent increase in the number of accidentrelated fatalities with 5286 deaths in 2016, 529 of which occurred in California, the second most of any state. The National Highway Traffic Safety Administration reports that 10 cars out of every 100,000 are involved in a fatal accident, while motorcycles have a fatality rate of 61 per 100,000. For every mile traveled, motorcyclists are 28 times more likely to be involved in a fatal accident [5]. 
Table 1 ASIA impairment scale grade and regional distribution by mechanism.

Extent of injury by ASIA impairment scale and regional distribution of injury by mechanism

\begin{tabular}{|c|c|c|c|c|c|c|c|}
\hline \multirow[t]{2}{*}{ Mechanism (Total $n=398$ ) } & \multicolumn{4}{|c|}{ ASIA impairment scale (\%) } & \multicolumn{3}{|c|}{ Regional distribution (\%) } \\
\hline & A & B & $\mathrm{C}$ & $\mathrm{D}$ & Cervical & Thoracic & Lumbar \\
\hline Bicycles $(n=41) 41 / 398=10 \%$ & 39 & 15 & 7 & 39 & 73 & 20 & 7 \\
\hline Motorcycles $(n=70) 70 / 398=18 \%$ & 56 & 13 & 8 & 23 & $37 *$ & $56^{*}$ & 7 \\
\hline Cars $(n=287) 287 / 398=72 \%$ & 53 & 12 & 13 & 22 & 68 & 28 & 4 \\
\hline
\end{tabular}

*Indicates statistical significance $(p<0.05)$.

While the correlation with motor vehicles and fatalities is well established, less is known about the patterns of SCIs in cars versus motorcycles, and understanding this information is critical in developing further safety measures. The present study set out to examine the extent of injury, patterns, and relative rates of occurrence of SCIs in cars versus motorcycles and bicycles in a population of adult trauma patients at a rehabilitation center in Los Angeles County, California.

\section{Materials and methods}

Rancho Los Amigos is a National Rehabilitation Center for SCI and the spine team reviews and consults with each SCI admission. SCI consults for a 10-year segment from 2003 to 2013 were selected and screened for a mechanism of injury involving a motor vehicular accident. All identified cases underwent chart review to determine neurological levels and extent of impairment, which were graded according to the International Standards for Neurological Classification of Spinal Cord Injury ISCoS [6]. Information gathered included date of injury, mechanism of injury (car, motorcycle, or bicycle), spinal region (cervical, thoracic, and lumbosacral) for the neurological level, and American Spinal Injury Association Impairment Scale (AIS) grade (A, B, C, D). All AIS grades were originally given in accordance with the most up-to-date standards at the time of exam and were not rescored as only minor revisions have occurred through time. By automobile or car, we mean the term inclusively to refer to all noncommercial four-wheeled passenger vehicles including sport utility vehicles, vans, minivans, and trucks. By motorcycle, we mean a motor driven vehicle with two in-line wheels. During data collection, non-motor driven bicycles emerged as a separate, but similar, mechanistic group and were included in the analysis.

For purposes of analysis, patients who sustained an AIS grade of A or B were grouped collectively in terms of extent of injury as "motor complete" and those with AIS C or D as "motor incomplete". Statistical analysis was performed using the Statistical Package for the Social Sciences Statistics 13 (IBM Corp, Armonk, NY) with $p$ value significance chosen at the $\alpha=0.05$ level. Categorical variables were analyzed using
Pearson chi-square test and Fisher exact test. Institutional Review Board approval was obtained.

\section{Results}

There were 398 SCIs over the 10-year period 2003-2013 that fit the inclusion criteria. The average age of the cohort was 37.6 years and $78.2 \%$ were male. Globally, the relative percentages of AIS A/B/C/D were similar across the three mechanisms and most injuries occurred at a spinal cord level, cervical or thoracic, with lumbar SCIs accounting for $<7 \%$ of the total (Table 1 ).

When stratified by spinal region, motorcycles, but not bicycles, had a significantly higher percentage of thoracic level SCIs as compared to autos (Table 1). Automobiles resulted in more cervical-level injury, as did bicycles. Thoracic SCIs, regardless of mechanism, were more likely motor complete than cervical or lumbar ones with a statistically significantly higher percentage of AIS A and B grades (Table 2).

\section{Discussion}

Most SCIs due to bicycle, motorcycle, or car accidents result in cervical or thoracic SCIs with lumbar levels accounting for $<7 \%$ of cases. Thoracic SCIs are both disproportionately more common in two-wheel motorcycle accidents as well as disproportionately more complete in AIS grade for all three mechanisms. Interestingly, unpowered two-wheel bicycles exhibit a pattern of SCI more similar to the pattern of four-wheel cars with a higher percentage of cervical than thoracic injuries compared to their two-wheel motorcycle counterparts.

When thoracic SCI occurs, it appears to be as an "all or nothing" phenomenon more than $80 \%$ of the time, regardless of the mechanism. By "all or nothing", we mean that either the thoracic spinal column gives way such that a greater extent of injury (AIS A/B) results or it does not and no SCI results. This makes biomechanical sense when considering the fact that the ribs and sternum largely constrain the less mobile thoracic spine. If a force is strong enough to break through a segment, 
Table 2 Extent of SCI by mechanism in terms of motor completeness.

\begin{tabular}{llll}
\hline & REGION & \multicolumn{2}{l}{ ASIA impairment scale } \\
\cline { 3 - 4 } & & $\begin{array}{l}\text { A/B (motor } \\
\text { complete) }(\%)\end{array}$ & $\begin{array}{l}\text { C/D (motor } \\
\text { incomplete) }(\%)\end{array}$ \\
\hline Bicycles & Cervical & 43 & 57 \\
$n=41$ & Thoracic & $88^{*}$ & 12 \\
& Lumbar & 67 & 33 \\
& Total & 54 & 46 \\
Motorcycles & Cervical & 58 & 42 \\
$n=70$ & Thoracic & $85^{*}$ & 15 \\
& Lumbar & 0 & 100 \\
Automobiles & Total & 69 & 31 \\
$n=287$ & Cervical & 61 & 39 \\
& Thoracic & $80^{*}$ & 20 \\
& Lumbar & 33 & 67 \\
& Total & 65 & 35 \\
\hline
\end{tabular}

*Indicates statistical significance $(p<0.05)$.

then there are few or no neighboring mobile segments to dissipate the remainder of that force to prevent displacement and significant cord damage. The thoracic canal also has slightly less canal space per segment than the cervical spine so even a smaller amount of displacement may be more significant [7]. The predominance of thoracic spinal cord injuries in motorcyclists is well documented. Robertson et al. found that 69 of $114(54.8 \%)$ spinal injuries in motorcyclists occurred in the thoracic segments [8]. They cited hyperflexion of the spine as the most common mechanism with axial loading concentrated at a point of maximal flexion, typically the mid-thoracic spine based on anatomic kyphosis, with $\mathrm{T} 6$ being the most common level [8]. In addition, a 1978 study examining SCI in motorcyclists demonstrated a $76 \%$ incidence of thoracic spine injury with $81 \%$ of these resulting in complete neurological injuries [9]. These data suggest that despite advances in safety equipment and legislation over four decades, these injury patterns have remained consistent.

For automobiles with lap and shoulder harnessing seat-belts restraining its passengers, a higher percentage of cervical SCIs compared to thoracolumbar SCIs seems anticipatable as the cervical spine is the primary region exposed the majority of the time aside from roll-over accidents, passenger ejections, or failure to use seat-belts. Reed et al. retrospectively examined spinal column injuries in order to compare patterns of injury between restrained and unrestrained passengers using the Abbreviated Injury Scale. They found that while serious thoracolumbar spine injuries (Abbreviated Injury Scale 3+) were significantly reduced with use of a seatbelt, serious cervical spine injuries (Abbreviated Injury Scale 3+) were not. However, when accounting for the combined effect of seatbelt and airbag, both were significantly reduced [10]. Interestingly, Fakharian et al. found that their survey of 304 patients involved in automobile accidents resulted in the majority suffering fractures in the lumbosacral region. However, in line with our findings, $83.3 \%$ of the patients with SCI were in the cervical region [11]. In the case of bicycles, it may be that more of these accidents occur at lower speeds so that the minimum threshold force to break through the thoracic spine is not as often attained as for motorcycles, even though both bicycles and motorcycles leave the cervical and thoracic spines largely unprotected during an accident as compared to automobiles. It may also be that larger coverage motorcycle helmets, on average, tend to be more protective of the cervical spine as compared to the average smaller coverage bicycle helmets, which may afford no particular cervical spine protection apart from its protection of the brain and head. Page et al. reviewed 1061 patients who had been injured in motorcycle crashes and treated at a single Level 1 trauma center over 5 years [12]. Of those patients, only $30.4 \%$ were wearing helmets. They found that cervical spine fractures occurred more often in patients who were not wearing helmets. Their findings support the idea that helmet use significantly reduces the likelihood of sustaining a cervical SCI during a motorcycle crash in addition to mitigating head injuries [12].

To estimate relative rates of occurrence of SCI for automobiles versus motorcycles, we compared our internal study percentages to the relative percentage of registered vehicles and valid driver's licenses in California, our home state. For motorcycles and automobiles (bicycles excluded), our data included 357 SCIs with motorcycles accounting for $20 \%$. For registered vehicles, in 2018, there were 857,677 motorcycles and 25,646,228 automobiles with motorcycles accounting for $3.3 \%$ of the total, while for valid $\mathrm{M}$ licenses to operate a motorcycle, there were only 1374 compared to $26,478,382$ for automobiles with motorcycle licenses accounting for only $0.005 \%$ of licensed drivers $[13,14]$. Using registered vehicle data, motorcycles were $20 \%$ of the SCIs we saw, but only $2.8 \%$ of the vehicles, suggesting motorcycle $\mathrm{SCI}$ incidence is 7 times higher than for cars $(20 \% / 2.8 \%)$. Using licensed persons, the SCI rate for motorcycles could be as high as 4000 times higher by extrapolation (20\%/0.005\%). Even if $90 \%$ of motorcycle riders were unlicensed riders, this would still suggest that SCI due to motorcycle is at least 400 times greater than that for automobiles. Though this estimate does not consider the actual miles driven per vehicle, which would be a truer denominator, nor the actual number of vehicles driven per day, the common-sense experience is that cars are driven, on average, relatively more often than motorcycles, particularly when considering motorcycles are driven less for inclement weather or when there are multiple passengers to transport. If true, any estimate would be an underestimate of the effect of motorcycle SCIs. Thus, motorcycles appear to have a far higher incidence of SCI compared to automobiles as well as a higher percentage of the more complete thoracic region injuries as determined directly from this study. 
Important limitations of this study include its retrospective nature, limits of the ability of researchers to search and accurately record data from consults, and the fact that consult information was occasionally incomplete. In addition, the data are susceptible to limitations in patient pool and referral patterns. Our institution does not discriminate SCI referrals based on mechanism or any other factor in accepting patients, but the population cared for tends to be under-served as part of a county safety-net hospital system, though not exclusively. All AIS grades were originally given in accordance with the most up-to-date standards at the time of exam. They were not rescored using the current standards upon gathering the data because the information available to us from the chart review was not complete enough to allow for revising the score. However, we do not feel that using the current standards would have significantly altered our results, as the modifications that have been made to the grading system are minimal when applied to our data.

\section{Conclusion}

Automobile, motorcycle, and bicycle SCIs occur primarily in the cervicothoracic cord region with lumbar SCI cases being rare $(<7 \%)$. Motorcycle SCIs have a higher predilection for the thoracic region, which are also more complete, on average, compared to their cervical counterparts. Bicycle SCIs follow automobiles with both having a higher percentage of cervical than thoracic SCIs compared to motorcycles. The overall relative rate of occurrence of SCI for motorcycles also appears to be much higher than for automobiles, likely 7-400 times higher, possibly more. This study hopes to help physicians anticipate and identify possible SCI in patients based on modes of transport and guide further research on finding means to reduce this risk. We also believe that it would be valuable to repeat this study using data from other countries to examine whether injury trends are similar and determine what variables may be responsible for any differences.

\section{Data availability}

The datasets generated and/or analyzed during the current study are available from the corresponding author on reasonable request.

\section{Compliance with ethical standards}

Conflict of interest The authors declare that they have no conflict of interest.

Publisher's note Springer Nature remains neutral with regard to jurisdictional claims in published maps and institutional affiliations.

\section{References}

1. Center for Disease Control and Prevention. Mortality in the United States. https://www.cdc.gov/nchs/products/databriefs/db267.htm. Accessed 12 Nov 2019.

2. Hatch BB, Wood-Wentz CM, Therneau TM, Walker MG, Payne JM, Reeves RK. Factors predictive of survival and estimated years of life lost in the decade following nontraumatic and traumatic spinal cord injury. Spinal Cord. 2017;55: $540-4$.

3. Hall OT, McGrath RP, Peterson MD, Chadd EH, DeVivo MJ, Heinemann AW, et al. The burden of traumatic spinal cord injury in the United States: disability-adjusted life years. Arch Phys Med Rehabil. 2019;100:95-100.

4. National Spinal Cord Injury Statistical Center. Facts and figures at a glance. https://www.nscisc.uab.edu. Accessed 12 November 2019.

5. National Highway Traffic Safety Administration. Traffic safety facts. https://crashstats.nhtsa.dot.gov/\#/DocumentTypeList/11. Accessed 12 November 2019.

6. Kirshblum SC, Burns SP, Biering-Sorensen F, Donovan W, Graves DE, Jha A, et al. International standards for neurological classification of spinal cord injury (Revised 2011). J Spinal Cord Med. 2011;34:535-46.

7. Frostell A, Hakim R, Thelin EP, Mattsson P, Svensson M. A review of the segmental diameter of the healthy human spinal cord. Front Neurol. 2016;7:238.

8. Robertson A, Giannoudis PV, Branfoot T, Barlow I, Matthews SJ, Smith RM. Spinal injuries in motorcycle crashes: patterns and outcomes. J Trauma. 2002;53:5-8.

9. Shrosbree RD. Spinal cord injuries of motorcycle accidents. Paraplegia. 1978;16:102-12.

10. Reed MA, Naftel RP, Carter S, MacLennan PA, McGwin G, Loring R. Motor vehicle restraint system use and risk of spine injury. Traffic Inj Prev. 2006;7:256-63.

11. Fakharian E, Mohammadzadeh M, Saberi HR, Fazel MR, Rejali $\mathrm{M}$, Akbari $\mathrm{H}$, et al. Spinal injury resulting from car accident: focus to prevention. Asian J Neurosurg. 2017;12:180-4.

12. Page PS, Wei Z, Brooks NP. Motorcycle helmets and cervical spine injuries: a 5-year experience at a level 1 trauma center. J Neurosurg Spine. 2018;28:607-11.

13. Department of Motor Vehicles. DMV statistics for publication. https://www.dmv.ca.gov/portal/dmv/detail/pubs/media_center/sta tistics. Accessed 12 Nov 2019.

14. Robertson A, Branfoot T, Barlow IF, Giannoudis PV. Spinal injury patterns resulting from car and motorcycle accidents. Spine. 2002;27:2825-30. 